\title{
Riboregulators: Fine-Tuning Virulence in Shigella
}

\author{
Megan E. Fris ${ }^{1}$ and Erin R. Murphy ${ }^{2 *}$ \\ ${ }^{1}$ Department of Biological Science, Ohio University, Athens, OH, USA, ${ }^{2}$ Department of Biomedical Sciences, Heritage \\ College of Osteopathic Medicine, Ohio University, Athens, OH, USA
}

Within the past several years, RNA-mediated regulation (ribo-regulation) has become increasingly recognized for its importance in controlling critical bacterial processes. Regulatory RNA molecules, or riboregulators, are perpetually responsive to changes within the micro-environment of a bacterium. Notably, several characterized riboregulators control virulence in pathogenic bacteria, as is the case for each riboregulator characterized to date in Shigella. The timing of virulence gene expression and the ability of the pathogen to adapt to rapidly changing environmental conditions is critical to the establishment and progression of infection by Shigella species; riboregulators mediate each of these important processes. This mini review will present the current state of knowledge regarding RNA-mediated regulation in Shigella by detailing the characterization and function of each identified riboregulator in these pathogens.

Keywords: riboregulator, Shigella, sRNA, pathogenesis, environment, small RNA

\section{INTRODUCTION}

\section{OPEN ACCESS}

Edited by:

William D. Picking,

University of Kansas, USA

Reviewed by:

Tim Yahr,

University of lowa, USA

Stephen Peter Kidd,

University of Adelaide, Australia

*Correspondence:

Erin R. Murphy murphye@ohio.edu

Received: 19 November 2015 Accepted: 08 January 2016 Published: 27 January 2016

Citation:

Fris ME and Murphy ER (2016) Riboregulators: Fine-Tuning Virulence in Shigella.

Front. Cell. Infect. Microbiol. 6:2. doi: 10.3389/fcimb.2016.00002
Shigella are bacterial pathogens highly adapted for colonizing the human gut, a process that is facilitated by their unique lifestyle. The bacteria are passed from host to host via the fecal oral route of transmission. After surviving the acidic environment of the stomach, Shigella species travel the length of the intestinal tract to the site of infection at the colonic epithelium (Jennison and Verma, 2003). Once at the colon, Shigella transverse the colonic epithelium by passage through M-cells and are subsequently presented to, and engulfed by, macrophages. Once inside the macrophage, Shigella species induces lysis of the phagocytic cell, releasing the bacteria to the basal-lateral surface of the epithelium. (Wassef et al., 1989) Next, Shigella invade human intestinal epithelial cells using a type three secretion system (TTSS) and begin to replicate within the eukaryotic cytoplasm. (Schroeder and Hilbi, 2008) Finally, the bacteria utilize host actin to spread from one eukaryotic cell to the next, a process that destroys intestinal epithelial cells thus contributing directly to the development of symptoms of a Shigella infection, namely bloody diarrhea (Jennison and Verma, 2003; Schroeder and Hilbi, 2008). The gene encoding any factor that directly or indirectly facilitates the ability of Shigella species to complete one or more process essential for pathogenesis must themselves be considered virulence-associated genes.

To establish and progress an efficient infection, Shigella species precisely regulate the expression of virulence-associated genes in response to specific environmental conditions encountered within the host; a collection of complex processes in which regulatory RNA molecules play critical, and ever increasingly recognized roles (Figure 1). Their ability to mediate a rapid, specific response makes riboregulators ideal molecules to mediate the regulation of virulence-associated gene expression in response to changes within a pathogen's micro-environment. Riboregulators characterized thus far in Shigella include several regulatory small RNAs (sRNAs) and one RNA 
thermometer (Murphy and Payne, 2007; Giangrossi et al., 2010; Gore and Payne, 2010; Africa et al., 2011; Tran et al., 2011; Broach et al., 2012; Kouse et al., 2013). Despite the fact that they function to regulate the expression of different target genes and that they utilize a variety of molecular mechanisms, all riboregulators described in Shigella to date share two important features; (1) they each respond, directly or indirectly, to changes in specific environmental conditions, and (2) they all are nestled within large regulatory networks that impact pathogenesis (Table 1 and Figure 1). This review will examine all characterized riboregulators in Shigella, with special emphasis placed on a discussion of how each was discovered, as well as their functions and impact on pathogenesis.

\section{CsrB AND CsrC}

Two sRNAs which are important regulatory molecules for carbon metabolism in E. coli are the carbon storage regulators CsrB and CsrC (Liu et al., 1997; Romeo, 1998; Weilbacher et al., 2003). CsrB and CsrC belong to a unique class of sRNAs which bind and sequester multiple copies of their target protein to inhibit its activity (Liu et al., 1997; Romeo, 1998; Weilbacher et al., 2003). In this case, CsrB and CsrC bind to and inhibit CsrA, a protein that promotes the production of other proteins necessary for glycolysis while inhibiting the production of proteins required for gluconeogenesis (Romeo et al., 1993; Sabnis et al., 1995; Yang et al., 1996; Liu et al., 1997; Weilbacher et al., 2003). CsrA also indirectly up-regulates the production of CsrB and CsrC through the UvrY/BarA two-component system, thus regulating

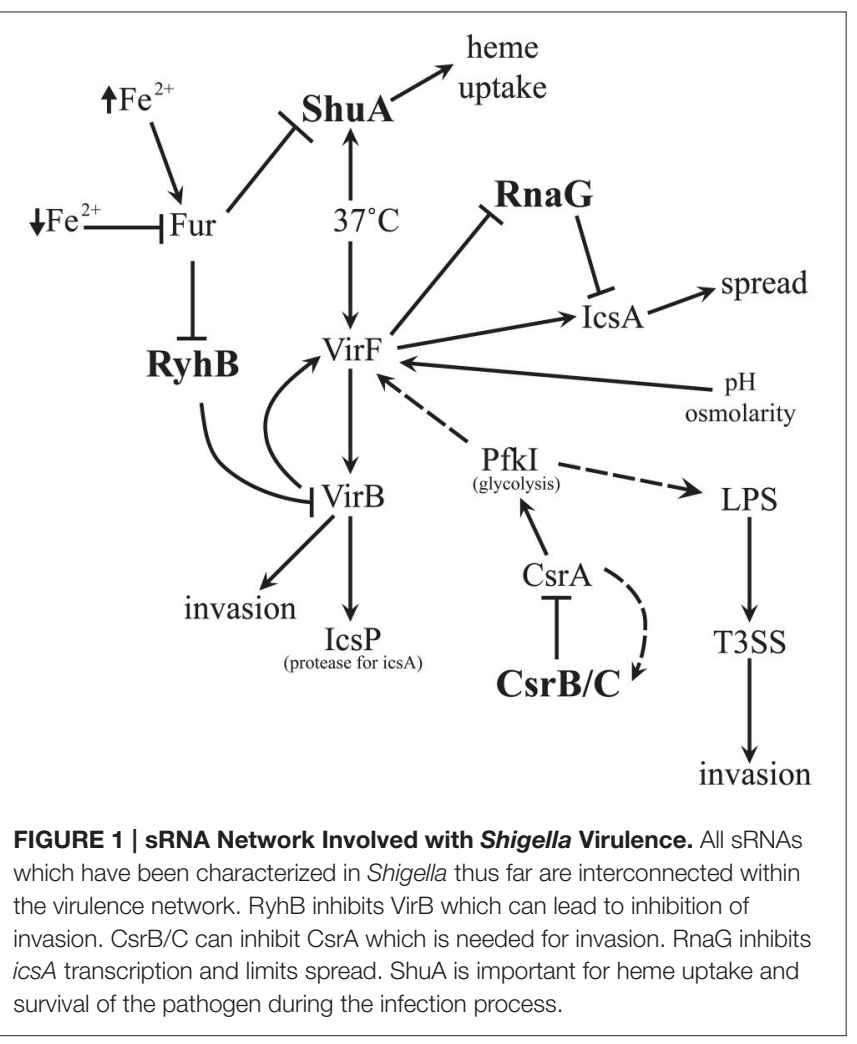

its own activity (Gudapaty et al., 2001; Suzuki et al., 2002). Key genes involved with carbon regulation in E. coli, including those encoding CsrA, CsrB, and CsrC as well as UvrY and BarA, are conserved in Shigella.

Interestingly, researchers have demonstrated that CsrA activity is linked to virulence in S. flexneri (Gore and Payne, 2010). Specifically, a mutation in $\operatorname{csr} A$ inhibits the ability of S. flexneri to invade eukaryotic cells and to spread from one eukaryotic cell to the next within a monolayer (Gore and Payne, 2010). Additionally a mutation in $\operatorname{csr} B$ or $\operatorname{csr} C$ which, in turn, indirectly increases the amount of free CsrA, allows S. flexneri to invade slightly more effectively than the wildtype strain (Gore and Payne, 2010). When CsrB or CsrC are over-produced, CsrA activity is inhibited as expected, and S. flexneri is no longer able to invade host cells (Gore and Payne, 2010). CsrA is hypothesized to impact Shigella virulence by two mechanisms. By the first mechanism, CsrA facilitates the activity of phosphofructokinase A (PfkA), which in turn upregulates production the master virulence regulator in Shigella, VirF (Adler et al., 1989; Gore and Payne, 2010). By the second mechanism, CsrA and PfkA may impact glycosylation of LPS on the surface of Shigella. Changes in LPS structure, mediated by alterations in the degree of glycosylation, impact the exposure of the type III secretion system (T3SS) on the surface of Shigella (West et al., 2005). Given these observations, changes in the Csr regulatory pathway could influence exposure of the Shigella T3SS (Hong and Payne, 1997; West et al., 2005; Gore and Payne, 2010). Regardless of the underlying molecular mechanism, CsrA clearly impacts pathogenesis in Shigella (Gore and Payne, 2010). As RNA molecules that function to regulate CsrA activity, CsrB and CsrC are thus implicated in the control of Shigella virulence.

Although different in size, no unique functions for CsrC and CsrB have been found thus far in either E. coli or Shigella. Investigating $\mathrm{CsrB}$ and $\mathrm{CsrC}$ under a number of different environmental could reveal unique activity and/or production patterns for these regulators (Weilbacher et al., 2003). In general,

TABLE 1 | Summary of Riboregulators in Shigella.

\begin{tabular}{lllll}
\hline Riboregulator & Type & $\begin{array}{l}\text { Environmental } \\
\text { influence }\end{array}$ & Target & $\begin{array}{l}\text { Virulence } \\
\text { associated } \\
\text { process }\end{array}$ \\
\hline CsrB/CsrC & $\begin{array}{l}\text { Sequestering } \\
\text { sRNAs }\end{array}$ & Carbon & CsrA & Invasion \\
RnaG & $\begin{array}{l}\text { Cis- } \\
\text { encoded }\end{array}$ & Temperature & icsA & Spread \\
& $\begin{array}{l}\text { sRNA } \\
\text { trans- } \\
\text { encoded } \\
\text { sRnA }\end{array}$ & Iron & VirB & Invasion \\
& $\begin{array}{l}\text { RNA } \\
\text { thermometer }\end{array}$ & $\begin{array}{l}\text { Iron and } \\
\text { temperature }\end{array}$ & ShuA & $\begin{array}{l}\text { Iron } \\
\text { acquisition } \\
\text { 5' UTR ShuA }\end{array}$ \\
& & & & from heme
\end{tabular}

Each riboregulator described in Shigella has also contributed to virulence of Shigella in some way. The sRNAs seem to effect invasion and spread while the RNA thermometer is important with iron acquisition. 
the biological significance of sibling sRNAs with apparent functional redundancies remains unclear (Caswell et al., 2014). It has been suggested that the extremely short half-life of CsrB and $\mathrm{CsrC}$ contributes to the speed by which the sRNAs can regulate $\mathrm{Csr} \mathrm{A}$, and that $\mathrm{CsrB}$ and $\mathrm{CsrC}$ allow for fine-tuning of gene expression in response to changes of carbon sources (Romeo, 1998). The short half-life of CsrB and CsrC could also contribute to the rapid responses needed for the regulation of pathogenesis based on environment specific alterations in carbon source availability.

\section{RyhB}

Another sRNA indirectly influenced by an environmental factor is RyhB. Originally discovered and characterized in E. coli, RyhB has been demonstrated to be an important regulator of iron metabolism (Massé and Gottesman, 2002). Bacteria need iron for survival, but too much iron can kill the organism, thus the production of iron uptake systems and iron storage systems are tightly regulated (Andrews et al., 2003). Studies in E. coli have demonstrated that RyhB plays an important role in maintaining the critical balance between the strict requirement and potential toxicity of iron (Massé and Gottesman, 2002; Andrews et al., 2003) Specifically, the production of RyhB itself is regulated in response to iron availability via the activity of the iron-responsive transcriptional regulator Fur (Bagg and Neilands, 1987; De Lorenzo et al., 1988; Hantke, 2001; Massé and Gottesman, 2002). Under conditions of high iron, Fur functions to inhibit RyhB production. Fur-dependent repression of RyhB production in turn relieves the RyhB-mediated repression of genes encoding various iron containing enzymes and iron storage proteins (Massé and Gottesman, 2002). RyhB is conserved between E. coli and Shigella where, as in E. coli, production of the sRNA is regulated by Fur, and activity of the sRNA impacts the expression of several targets conserved between the two genus (Murphy and Payne, 2007).

In addition to the role that RyhB plays in iron metabolism, RyhB has been implicated in the regulation of virulenceassociated gene expression in S. dysenteriae (Murphy and Payne, 2007; Africa et al., 2011; Broach et al., 2012). Specifically, RyhB inhibits the transcription of $\operatorname{vir} B$, a gene encoding a protein that functions to promote the expression of several virulenceassociated genes in Shigella. (Adler et al., 1989; Beloin et al., 2002; Murphy and Payne, 2007; Africa et al., 2011; Kane and Dorman, 2012). Although the exact molecular mechanism underlying RyhB-dependent inhibition of virB transcription remains unknown, complementarity between the template DNA strand within the virB open reading frame and RyhB exists, and is required for the observed regulation; data that suggests a novel regulatory mechanism may be at play (Broach et al., 2012).

RyhB allows for iron responsive regulation of the Shigella virulence cascade. In the relatively iron-rich environment of the human gut, Fur is likely active and functioning to repress the production of RyhB. With decreased production of RyhB, VirB production proceeds and the protein functions to promote the expression of several virulence-associated genes including ics $P$ (Wing et al., 2004; Castellanos et al., 2009; Broach et al., 2012).
IcsP protease limits IcsA (a protein required to polymerize the actin tail used by Shigella to spread from one eukaryotic cell to the next) from being produced prior to invasion into the host cell (Makino et al., 1986; Bernardini et al., 1989; Lett et al., 1989; Goldberg and Theriot, 1995; Egile et al., 1997; Shere et al., 1997; Steinhauer et al., 1999; Wing et al., 2005; Africa et al., 2011). Once Shigella enter the host cell, iron conditions become limiting, and as a result Fur-mediated repression of RyhB production is relieved. Once produced in the low iron environment RyhB functions to represses $v i r B$ expression, which in turn limits IcsP production (Wing et al., 2004; Africa et al., 2011). Decreased IcsP production results in increased activity of IcsA which in turn facilitates host actin polymerization and cell to cell spreading by the bacterium (Makino et al., 1986; Bernardini et al., 1989; Lett et al., 1989; Goldberg et al., 1993; Goldberg and Theriot, 1995).

Inhibition of $\operatorname{vir} B$ transcription is not the only way by which RyhB influences pathogenesis in Shigella. In addition to its role in modulating VirB production, RyhB also indirectly regulates the expression of genes encoding factors required for acid resistance, an essential aspect of infection initiation by this pathogen (Oglesby et al., 2005).

Evolutionarily, as an sRNA RyhB is likely a more adapt regulator than a protein would be. To compensate for small changes in iron availability within the human host, the synthesis of RyhB can quickly be inhibited by Fur. RyhB can also become a fully active regulator after only transcription, giving it an edge over a protein regulator which would require more energy and time to synthesize (Beisel and Storz, 2010). Its fundamental features as an iron-regulated ribo-regulator allow for quick, efficient modulation of target gene expression by RyhB in response to the subtle changes of environmental iron availability experienced by the pathogen throughout the course of a natural infection.

\section{RnaG}

RnaG is unique among Shigella sRNA in that, unlike the others, it was first identified and characterized in Shigella, and it is encoded on the large virulence plasmid. Similar to other Shigella sRNAs however, is the fact that production of $\mathrm{RnaG}$ is regulated in response to a specific environmental cue and that once produced, it functions to impact pathogenesis. Specifically, RnaG production is indirectly controlled in response to environmental temperature, and once produced functions to regulate the transcription of $i c s A$, a virulence-associated gene required for actin-based motility of Shigella species (Bernardini et al., 1989; Giangrossi et al., 2010; Tran et al., 2011). Two coordinated mechanisms allow RnaG to mediate transcriptional control of $i c s A$. First, $r n a G$ and ics $A$ have convergent promoters in close proximity to each other (Giangrossi et al., 2010). As such, activity of the $r n a G$ promoter results in decreased activity of the $i c s A$ promoter through promoter interference (Giangrossi et al., 2010). Second, as a result of their over-lapping arrangement, and thus nucleic acid complementarity, RnaG can bind directly to the icsA transcript via kissing complexes, alter the structure of the growing transcript, and lead to early transcriptional 
termination (Giangrossi et al., 2010). Through these two, nonmutually exclusive molecular mechanisms, transcription of the important virulence factor IcsA is inhibited by RnaG, thus directly implicating this small RNA in controlling Shigella pathogenesis.

RnaG is likely produced during the time Shigella first enters the host until the pathogen reaches the site of infection. During this time, RnaG would inhibit premature expression of ics $A$, thus preventing the production of proteins required for host actin polymerization (Giangrossi et al., 2010). At $37^{\circ} \mathrm{C}$ (the environmental temperature within the host), $\mathrm{VirF}$ is produced and functions to promote the transcription of ics $A$, thus inhibiting that of $r n a G$ (Tran et al., 2011). During the initial stages of infection, however, it is possible that VirF levels are not high enough to induce ics $A$ transcription to levels required for efficient actin polymerization and, due to specific environmental factors such as $\mathrm{pH}$ and osmolarity, may not be high enough until Shigella reaches the colonic epithelium (Porter and Dorman, 1994; Nakayama and Watanabe, 1995; Kane and Dorman, 2012). In this case, RnaG production during these initial stages of infection would inhibit aberrant icsA expression between the time that Shigella first enters the host and when the pathogen reaches the site of infection. Such temporal timing would prevent premature production of IcsA and possibly damper any immune system alarms which may be set off in the presence of the protein.

\section{ShuA}

The final Shigella ribo-regulator is an RNA thermometer located within the 5' untranslated region (UTR) of S. dysenteriae shuA (Shigella heme uptake), a gene encoding an outer-membrane heme receptor that is essential for the utilization of heme as a source of nutrient iron by the pathogen (Mills and Payne, 1995, 1997). RNA thermometers function to modulate translation efficiency from the transcript on which they are housed by the formation of an inhibitory structure(s) that physically blocks binding of the ribosome to the transcript at non-permissive (low) temperatures (Kortmann and Narberhaus, 2012). As the environmental temperature rises the inhibitory structure is destabilized, the ribosomal binding site is exposed and translation of the regulated gene proceeds. The shuA RNA thermometer represents the first RNA thermometer characterized in any Shigella species (Kouse et al., 2013). Although identified initially in Shigella, an identical regulator functions to control expression of the orthologous gene chuA in pathogenic E. coli where this gene product is a virulence determinant (Wyckoff et al., 1998; Hoffmann et al., 2001; Torres et al., 2001; Okeke et al., 2004). Transcription of $s h u A$ is subject to iron-dependent regulation by the protein Fur while translation from the shuA transcript is subject to temperature-dependent regulation by the activity of the cis-encoded RNA thermometer (Mills and Payne, 1995, 1997; Kouse et al., 2013). It is important to note, that for bacterial pathogens increased environmental temperature can act as an important signal that the organism has entered the host, the environment where production of virulence-associated factors will provide the most benefit.
Only under particular environmental conditions will ShuA be efficiently produced. Under conditions where iron is abundant, shuA transcription will be repressed by the activity of Fur, regardless of environmental temperature. Conditions where iron is depleted, but the environmental temperature is relatively low, the FourU RNA thermometer will inhibit translation of shuA. Only in iron-limiting and at temperatures corresponding to that within the human body $\left(37^{\circ} \mathrm{C}\right)$, will ShuA be produced (Kouse et al., 2013). The transcriptional and translation regulation mediated by Fur and the shuA RNA thermometer, respectively, function together to ensure maximal production of ShuA under conditions of poor iron availability and increased temperature, precisely the condition encountered within the human body; the only environment in which Shigella will encounter heme as a potential source of essential nutrient iron.

\section{DISCUSSION}

The riboregulators in Shigella, described in this review, all respond (directly or indirectly) to environmental changes, and all of them function within larger regulatory networks to influence pathogenesis of these species. CsrB/CsrC are regulated in response to carbon availability, RnaG is regulated in response to temperature, $\mathrm{RyhB}$ is regulated in response to iron availability, and finally the activity of the shuA RNA thermometer is regulated in response to temperature. Importantly, every Shigella ribo-regulator characterized to date functions to influence the production of factors involved in one or more processes required for pathogenesis, and thus must themselves be considered virulence determinants. This observation raises the question, why are some virulence-associated processes in Shigella controlled by protein-based regulation while others are controlled, at least in part, by the activity of riboregulators? In all sRNA found in Shigella thus far, proteins (VirF, H-NS, Fur, and UvrY) seem to be the initial regulator controlling a given step of a specific virulence-associated process. In each case, the ribo-regulator functions to modulate a specific virulence-associated activity for some duration of time, and then due to an environmental trigger, quickly switches off and allows Shigella to proceed to the next stage of pathogenesis (Figure 1). Perhaps Shigella evolved to favor riboregulators over protein regulators in conditions under which rapid specific changes to the production of one or just a few genes would be more beneficial to the organism than turning on/off an entire large regulon. Perhaps, processes required for the initial induction of pathogenesis in Shigella is controlled by protein regulators rather than riboregulators because quick reactions to false positive signals for pathogenesis could be detrimental to the survival of the bacteria, while a lag in protein regulation may temper those signals, thus reducing the frequency of such detrimental events. (Beisel and Storz, 2010).

More research needs to be done on riboregulators in Shigella to fully understand their functions and roles in virulence regulation (Storz et al., 2011). Such studies are likely to be fueled by genomic-based analyses that suggest the presence of additional ribo-regulators in Shigella, regulators for which function(s) have not yet been elucidated (Peng et al., 2011; Skippington and Ragan, 
2012). Additionally, many putative sRNAs should be examined to see if they have small proteins missed by predictor programs (Storz et al., 2014). Lastly, once sRNAs and riboregulators are fully understood, it is possible that their function could be targeted as novel treatments for shigellosis.

\section{AUTHOR CONTRIBUTIONS}

Wrote the article: MF and EM. Edited the article: MF and EM. Created the figure: MF. Created the table: MF.

\section{REFERENCES}

Adler, B., Sasakawa, C., Tobe, T., Makino, S., Komatsu, K., and Yoshikawa, M. (1989). A dual transcriptional activation system for the $230 \mathrm{~kb}$ plasmid genes coding for virulence-associated antigens of Shigella flexneri. Mol. Microbiol. 3, 627-635. doi: 10.1111/j.1365-2958.1989.tb00210.x

Africa, L. A. A., Murphy, E. R., Egan, N. R., Wigley, A. F., and Wing, H. J. (2011). The Iron-Responsive Fur/RyhB regulatory cascade modulates the Shigella outer membrane protease IcsP. Infect. Immun. 79, 4543-4549. doi: 10.1128/IAI.05340-11

Andrews, S. C., Robinson, A. K., and Rodríguez-Quiñones, F. (2003). Bacterial iron homeostasis. FEMS Microbiol. Rev. 27, 215-237. doi: 10.1016/S01686445(03)00055-X

Bagg, A., and Neilands, J. B. (1987). Ferric uptake regulation protein acts as a repressor, employing iron (II) as a cofactor to bind the operator of an iron transport operon in Escherichia coli. Biochemistry 26, 5471-5477. doi: 10.1021/bi00391a039

Beisel, C. L., and Storz, G. (2010). Base pairing small RNAs and their roles in global regulatory networks. FEMS Microbiol. Rev. 34, 866-882. doi: 10.1111/j.15746976.2010.00241.x

Beloin, C., McKenna, S., and Dorman, C. J. (2002). Molecular dissection of VirB, a key regulator of the virulence cascade of Shigella flexneri. J. Biol. Chem. 277, 15333-15344. doi: 10.1074/jbc.M111429200

Bernardini, M. L., Mounier, J., d'Hauteville, H., Coquis-Rondon, M., and Sansonetti, P. J. (1989). Identification of icsA, a plasmid locus of Shigella flexerni that governs bacterial intra- and intracellular spread thorugh interaction with F-actin. Proc. Natl. Acad. Sci. U.S.A. 86, 3867-3871.

Broach, W. H., Egan, N., Wing, H. J., Payne, S. M., and Murphy, E. R. (2012). VirF-independent regulation of Shigella virB transcription is mediated by the small RNA RyhB. PLOS ONE 7:e38592. doi: 10.1371/journal.pone. 0038592

Castellanos, M. I., Harrison, D. J., Smith, J. M., Labahn, S. K., Levy, K. M., and Wing, H. J. (2009). VirB alleviates H-NS repression of the icsP promoter in Shigella flexneri from sites more than one kilobase upstream of the transcription start site. J. Bacteriol. 191, 4047-4050. doi: 10.1128/JB.00313-09

Caswell, C. C., Oglesby-Sherrouse, A. G., and Murphy, E. R. (2014). Sibling rivalry: related bacterial small RNAs and their redundant and non-redundant roles. Front. Cell. Infect. Microbiol. 4:151. doi: 10.3389/fcimb.2014.00151

De Lorenzo, V., Giovannini, F., Herrero, M., and Neilands, J. B. (1988). Metal ion regulation of gene expression fur repressor-operator interaction at the promoter region of the aerobactin system of pColV-K30. J. Mol. Biol. 203, 875-884. doi: 10.1016/0022-2836(88)90113-1

Egile, C., d'Hauteville, H., Parsot, C., and Sansonetti, P. J. (1997). SopA, the outer membrane protease responsible for polar localization of IcsA in Shigella flexneri. Mol. Microbiol. 23, 1063-1073.

Giangrossi, M., Prosseda, G., Tran, C. N., Brandi, A., Colonna, B., and Falconi, M. (2010). A novel antisense RNA regulates at transcriptional level the virulence gene icsA of Shigella flexneri. Nucleic Acids Res. 38, 3362-3375. doi: 10.1093/nar/gkq025

Goldberg, M. B., Bârzu, O., Parsot, C., and Sansonetti, P. J. (1993). Unipolar localization and ATPase activity of IcsA, a Shigella flexneri protein involved in intracellular movement. J. Bacteriol. 175, 2189-2196.

\section{FUNDING}

Studies in the Murphy lab have been supported by the National Institutes of Health, the American Heart Association, Ohio University, and Ohio University College of Osteopathic Medicine.

\section{ACKNOWLEDGMENTS}

We would like to thank Ohio University and the National Institute of Health (R15AI103887-01A1).

Goldberg, M. B., and Theriot, J. A. (1995). Shigella flexneri surface protein IcsA is sufficient to direct actin-based motility. Proc. Natl. Acad. Sci. U.S.A. 92, 6572-6576. doi: 10.1073/pnas.92.14.6572

Gore, A. L., and Payne, S. M. (2010). CsrA and Cra influence Shigella flexneri pathogenesis. Infect. Immun. 78, 4674-4682. doi: 10.1128/IAI.00 589-10

Gudapaty, S., Suzuki, K., Wang, X., Romeo, T., Wang, X. I. N., and Babitzke, P. (2001). Regulatory Interactions of Csr Components: the RNA binding protein CsrA activates csrB transcription in Escherichia coli. J. Bacteriol. 183, 6017-6027. doi: 10.1128/JB.183.20.6017-6027.2001

Hantke, K. (2001). Iron and metal regulation in bacteria. Curr. Opin. Microbiol. 4, 172-177. doi: 10.1016/S1369-5274(00)00184-3

Hoffmann, H., Hornef, M. W., Schubert, S., and Roggenkamp, A. (2001). Distribution of the outer membrane haem receptor protein ChuA in environmental and human isolates of Escherichia coli. Int. J. Med. Microbiol. 291, 227-230. doi: 10.1078/1438-4221-00123

Hong, M., and Payne, S. M. (1997). Effect of mutations in Shigella flexneri chromosomal and plasmid-encoded lipopolysaccharide genes on invasion and serum resistance. Mol. Microbiol. 24, 779-791. doi: 10.1046/j.13652958.1997.3731744.x

Jennison, A. V., and Verma, N. K. (2003). Shigella flexneri infection: pathogenesis and vaccine development. FEMS Microbiol. Rev. 28, 43-58. doi: 10.1016/j.femsre.2003.07.002

Kane, K. A., and Dorman, C. J. (2012). VirB-mediated positive feedback control of the virulence gene regulatory cascade of Shigella flexneri. J. Bacteriol. 194, 5264-5273. doi: 10.1128/JB.00800-12

Kortmann, J., and Narberhaus, F. (2012). Bacterial RNA thermometers: molecular zippers and switches. Nat. Rev. Microbiol. 10, 255-265. doi: 10.1038/nrmicro2730

Kouse, A. B., Righetti, F., Kortmann, J., Narberhaus, F., and Murphy, E. R. (2013). RNA-mediated thermoregulation of iron-acquisition genes in Shigella dysenteriae and pathogenic Escherichia coli. PLOS ONE 8:e63781. doi: 10.1371/journal.pone.0063781

Lett, M.-C., Sasakawa, C., Okada, N., Sakai, T., Makino, S., Yamada, M., et al. (1989). virG, a plasmid-coded virulence gene of Shigella flexneri: identification of the virG protein and determination of the complete coding sequence. J. Bacteriol. 171, 353-359. Available online at: http://jb.asm.org/content/171/ 1/353.long

Liu, M. Y., Gui, G., Wei, B., Preston, J. F. III., Oakford, L., Yuksel, U., et al. (1997). The RNA molecule CsrB binds to the global reguatory protein CsrA and antagonizes its activity in Escherichia coli. J. Biol. Chem. 272, 17502-17510. doi: $10.1074 /$ jbc.272.28.17502

Makino, S., Sasakawa, C., Kamata, K., Kurata, T., and Yoshikawa, M. (1986). A genetic determinant required for gontinuous reinfection of adjacent cells on large plasmid in S. flexneri 2a. Cell 46, 551-555. doi: 10.1016/00928674(86)90880-9

Massé, E., and Gottesman, S. (2002). A small RNA regulates the expression of genes involved in iron metabolism in Escherichia coli. Proc. Natl. Acad. Sci. U.S.A. 99, 4620-4625. doi: 10.1073/pnas.032066599

Mills, M., and Payne, S. M. (1995). Genetics and regulation of heme iron transport in Shigella dysenteriae and detection of an analogous system in Escherichia coli O157: H7. J. Bacteriol. 177, 3004-3009. 
Mills, M., and Payne, S. M. (1997). Identification of shuA, the gene encoding the heme receptor of Shigella dysenteriae, and analysis of invasion and intracellular multiplication of a shuA mutant. Infect. Immun. 65, 5358-5363.

Murphy, E. R., and Payne, S. M. (2007). RyhB, an iron-responsive small RNA molecule, regulates Shigella dysenteriae virulence. Infect. Immun. 75, 3470-3477. doi: 10.1128/IAI.00112-07

Nakayama, S., and Watanabe, H. (1995). Involvement of $c p x A$, a sensor of a twocomponent regulatory system, in the $\mathrm{pH}$-dependent regulation of expression of Shigella sonnei virF gene. J. Bacteriol. 177, 5062-5069.

Oglesby, A. G., Murphy, E. R., Iyer, V. R., and Payne, S. M. (2005). Fur regulates acid resistance in Shigella flexneri via RyhB and ydeP. Mol. Microbiol. 58, 1354-1367. doi: 10.1111/j.1365-2958.2005.04920.x

Okeke, I. N., Scaletsky, I. C. A., Soars, E. H., Macfarlane, L. R., and Torres, A. G. (2004). Molecular epidemiology of the iron utilization genes of enteroaggregative Escherichia coli. J. Clin. Microbiol. 42, 36-44. doi: 10.1128/JCM.42.1.36-44.2004

Peng, J., Yang, J., and Jin, Q. (2011). An Integrated approach for finding overlooked genes in Shigella. PLoS ONE 6:e18509. doi: 10.1371/journal.pone.0018509

Porter, M. E., and Dorman, C. J. (1994). A role for H-NS in the thermo-osmotic regulation of virulence gene expression in Shigella flexneri. J. Bacteriol. 176, 4187-4191.

Romeo, T. (1998). Global regulation by the small RNA-binding protein CsrA and the non- coding RNA molecule CsrB. Mol. Microbiol. 29, 1321-1330. doi: 10.1046/j.1365-2958.1998.01021.x

Romeo, T., Gong, M., Liu, M. Y., and Brun-Zinkernagel, A.-M. (1993). Identification and molecular characterization of $\operatorname{csr} A$, a pleiotropic gene from Escherichia coli that affects glycogen biosynthesis, gluconeogenesis, cell size, and surface properties. J. Bacteriol. 175, 4744-4755.

Sabnis, N. A., Yang, H., and Romeo, T. (1995). Pleiotropic regulation of central carbohydrate metabolism in Escherichia coli via the gene csrA. J. Biol. Chem. 270, 29096-29104. doi: 10.1074/jbc.270.49.29096

Schroeder, G. N., and Hilbi, H. (2008). Molecular pathogenesis of Shigella spp.: controlling host cell signaling, invasion, and death by type III secretion. Clin. Microbiol. Rev. 21, 134-156. doi: 10.1128/CMR.00032-07

Shere, K. D., Sallustio, S., Manessis, A., D’Aversa, T. G., and Goldberg, M. B. (1997). Disruption of IcsP, the major Shigella protease that cleaves IcsA, accelerates actin-based motility. Mol. Microbiol. 25, 451-462. doi: 10.1046/j.1365-2958.1997.4681827.x

Skippington, E., and Ragan, M. A. (2012). Evolutionary dynamics of small RNAs in 27 Escherichia coli and Shigella Genomes. Genome Biol. Evol. 4, 330-345. doi: 10.1093/gbe/evs001

Steinhauer, J., Agha, R., Pham, T., Varga, A. W., and Goldberg, M. B. (1999). The unipolar Shigella surface protein IcsA is targeted directly to the bacterial old pole: IcsP cleavage of IcsA occurs over the entire bacterial surface. Mol. Microbiol. 32, 367-377. doi: 10.1046/j.1365-2958.1999.01356.x

Storz, G., Vogel, J., and Wassarman, K. M. (2011). Regulation by small RNAs in bacteria: expanding frontiers. Mol. Cell 43, 880-891. doi: 10.1016/j.molcel.2011.08.022

Storz, G., Wolf, Y. I., and Ramamurthi, K. S. (2014). Small proteins can no longer be ignored. Annu. Rev. Biochem. 83, 753-777. doi: 10.1146/annurev-biochem070611-102400
Suzuki, K., Wang, X., Weilbacher, T., Pernestig, A.-K., Melefors, O., Georgellis, D., et al. (2002). Regulatory circuitry of the CsrA / CsrB and BarA / UvrY systems of Escherichia coli. J. Bacteriol. 184, 5130-5140. doi: 10.1128/JB.184.18.51305140.2002

Torres, A. G., Redford, P., Welch, R. A., and Payne, S. M. (2001). TonB-dependent systems of uropathogenic Escherichia coli: aerobactin and heme transport and TonB are required for virulence in the mouse. Infect. Immun. 69, 6179-6185. doi: 10.1128/IAI.69.10.6179-618 5.2001

Tran, C. N., Giangrossi, M., Prosseda, G., Brandi, A., Di Martino, M. L., Colonna, B., et al. (2011). A multifactor regulatory circuit involving H-NS, VirF and an antisense RNA modulates transcription of the virulence gene icsA of Shigella flexneri. Nucleic Acids Res. 39, 8122-8134. doi: 10.1093/nar/g $\operatorname{kr} 521$

Wassef, J. S., Keren, D. F., and Mailloux, J. L. (1989). Role of M cells in initial antigen uptake and in ulcer formation in the rabbit intestinal loop model of shigellosis. Infect. Immun. 57, 858-863.

Weilbacher, T., Suzuki, K., Dubey, A. K., Wang, X., Gudapaty, S., Morozov, I., et al. (2003). A novel sRNA component of the carbon storage regulatory system of Escherichia coli. Mol. Microbiol. 48, 657-670. doi: 10.1046/j.13652958.2003.03459.x

West, N. P., Sansonetti, P., Mounier, J., Exley, R. M., Parsot, C., Guadagnini, S., et al. (2005). Optimization of virulence functions through glucosylation of Shigella LPS. Science 307, 1313-1317. doi: 10.1126/science.11 08472

Wing, H. J., Goldman, S. R., Ally, S., and Goldberg, M. B. (2005). Modulation of an outer membrane protease contributes to the virulence defect of Shigella flexneri Strains carrying a mutation in the virK locus. Infect. Immun. 73, 1217-1220. doi: 10.1128/IAI.73.2.1217-1220.2005

Wing, H. J., Yan, A. W., Goldman, S. R., and Goldberg, M. B. (2004). Regulation of IcsP, the outer membrane protease of the Shigella actin tail assembly protein IcsA, by virulence plasmid regulators VirF and VirB. J. Bacteriol. 186, 699-705. doi: 10.1128/JB.186.3.699-705.2004

Wyckoff, E. E., Duncan, D., Torres, A. G., Mills, M., Maase, K., and Payne, S. M. (1998). Structure of the Shigella dysenteriae haem transport locus and its phylogenetic distribution in enteric bacteria. Mol. Microbiol. 28, 1139-1152. doi: 10.1046/j.1365-2958.1998.00873.x

Yang, H., Liu, M. Y., and Romeo, T. (1996). Coordinate genetic regulation of glycogen catabolism and biosynthesis in Escherichia coli via the CsrA gene product. J. Bacteriol. 178, 1012-1017.

Conflict of Interest Statement: The authors declare that the research was conducted in the absence of any commercial or financial relationships that could be construed as a potential conflict of interest.

Copyright (c) 2016 Fris and Murphy. This is an open-access article distributed under the terms of the Creative Commons Attribution License (CC BY). The use, distribution or reproduction in other forums is permitted, provided the original author(s) or licensor are credited and that the original publication in this journal is cited, in accordance with accepted academic practice. No use, distribution or reproduction is permitted which does not comply with these terms. 